\title{
BRAF mutations in thyroid tumors from an ethnically diverse group
}

\author{
Hans-Juergen Schulten ${ }^{1 *}$, Sherine Salama ${ }^{2}$, Zuhoor Al-Mansouri ${ }^{3}$, Reem Alotibi ${ }^{4}$, Khalid Al-Ghamdi ${ }^{5}$, \\ Osman Abdel Al-Hamour ${ }^{6}$, Hassan Sayadi ${ }^{3}$, Hosam Al-Aradati ${ }^{3}$, Adel Al-Johari ${ }^{5}$, Etimad Huwait ${ }^{4}$, Mamdooh Gari ${ }^{1}$, \\ Mohammed Hussain Al-Qahtani ${ }^{1}$ and Jaudah Al-Maghrabi ${ }^{2,3}$
}

\begin{abstract}
Background: The molecular etiology of thyroid carcinoma (TC) and other thyroid diseases which may present malignant precursor lesions is not fully explored yet. The purpose of this study was to estimate frequency, type and clinicopathological value of BRAF exon 15 mutations in different types of cancerous and non-cancerous thyroid lesions originating in an ethnically diverse population.

Methods: BRAF exon 15 was sequenced in 381 cases of thyroid lesions including Hashimoto's thyroiditis, nodular goiters, hyperplastic nodules, follicular adenomas (FA), papillary TC (PTC), follicular variant PTC (FVPTC), microcarcinomas of PTC (micro PTC; tumor size $\leq 1 \mathrm{~cm}$ ), follicular TC (FTC), and non-well differentiated TC (non-WDTC).
\end{abstract}

Results: We identified BRAF mutations in one of 69 FA, 72 of 115 (63\%) PTC, seven of 42 (17\%) FVPTC, 10 of 56 (18\%) micro PTC, one of 17 (6\%) FTC, and one of eight (13\%) non-WDTC. Most of the cases showed the common V600E mutation. One case each of PTC, FVPTC, and FTC harbored a K601E mutation. A novel BRAF mutation was identified in a FA leading to deletion of threonine at codon 599 (p.T599del). A rare 3-base pair insertion was detected in a stage III PTC resulting in duplication of threonine at codon 599 (p.T599dup). Patients with PTC harboring no BRAF mutation (BRAF ${ }^{\mathrm{wt}}$ ) were on average younger than those with a BRAF mutation (BRAF ${ }^{\text {mut }}$ ) in the PTC (36.6 years vs. 43.8 years). Older age ( $\geq 45$ years) in patients with PTC was significantly associated with tumor size $\geq 4 \mathrm{~cm}(P=0.018)$, vessel invasion $(P=0.004)$, and distant metastasis $(P=0.001)$. Lymph node $(L N)$ involvement in PTC significantly correlated with tumor size $(P=0.044)$, and vessel invasion $(P=0.013)$. Of notice, taken the whole TC group, family history of thyroid disease positively correlated with capsular invasion ( $P=0.025)$.

Conclusions: Older age is manifold associated with unfavorable tumor markers in our series. The K601E identified in a PTC, FVPTC, and FTC seems to be more distributed among different histological types of TC than previously thought. The T599del is a yet undescribed mutation and the rare T599dup has not been reported as a mutation in PTC so far.

Keywords: BRAF mutations, Deletion codon 599, Duplication codon 599, Thyroid carcinoma, Follicular adenoma, Family history, MENA region

\footnotetext{
* Correspondence: hschulten@kau.edu.sa

${ }^{1}$ Center of Excellence in Genomic Medicine Research, King Abdulaziz University, Jeddah, Saudi Arabia

Full list of author information is available at the end of the article
} 


\section{Background}

TC represents a considerable cancer burden worldwide [1,2]. In Saudi Arabia it is considered as the fourth most common cancer type with $6.4 \%$ of all diagnosed cancers [3]. Median age of women diagnosed with TC in Saudi Arabia is considerable lower than for men (37 years $v$ s. 43 years). Notably, females in this country are more commonly diagnosed with FVPTC (19.3\% vs. 9.6\%) and micro PTC (7.4\% vs. 2.6\%) than males.

Differentiated types of TC arising from endodermally derived follicular cells comprise papillary TC (PTC), follicular TC (FTC), and Hurthle cell carcinoma. Hurthle cell carcinomas are also considered as a subtype of FTC. Anaplastic TC (ATC) is considered as undifferentiated TC which either may evolve de novo or from PTC or FTC [4]. Medullary TC (MTC) are derived from calcitonin-producing $\mathrm{C}$ cells and own a different etiology involving commonly mutations in the RET oncogene. PTC represents about $80 \%$ of differentiated TC with an increasing trend while FTC ( $11 \%)$, Hurthle cell carcinoma $(\sim 3 \%)$, MTC ( 4\%), and ATC ( 2\%) represent minor variants [2]. A study on the pattern of TC arising in Jeddah, an ethnically diverse metropolis in Western Saudi Arabia, revealed a common distribution of histological types: PTC (82\%), FTC (4.4\%), ATC (6.7\%), and MTC (6.7\%) [5].

The pathogenesis of the majority of TC is affected by somatic mutations or rearrangements in known TC genes. The $\mathrm{BRAF}$ gene is the most frequently mutated gene in TC. BRAF is a cytoplasm receptor serine/threonine kinase and a key molecule in the mitogen activated protein kinase (MAPK) pathway. It is mutated in diverse human malignancies although the frequency varies significantly between different types of cancers [6]. In malignant melanomas and in TC, which both are oncogenic transformations of neural crest derived cell lineages, frequency of BRAF mutations can exceed $80 \%$ of the cases [6,7]. BRAF activation affects in the vast majority of cases codon 600 located within the regulatory domain encoded by exon 15. Most common mutation at codon 600 is a valine-to-glutamic acid transversion (V600E). This mutation disrupts the inactive conformation of BRAF as it mimics activational phosphorylation at the adjacent residues and results in constitutive downstream signaling [8]. Other BRAF activation mutations are far less common in TC like the K601E mutation that is commonly restricted to FVPTC [9]. Although the prognostic impact of BRAF mutations in PTC is controversially discussed, the V600E mutation seems to be a valid target for molecular therapy as this mutation confers elevated resistance to iodine ablation $[7,10]$. We assessed the impact of BRAF mutations in cancerous and non-cancerous thyroid lesions originating in an ethnically diverse population by a comprehensive mutational survey.

\section{Methods}

\section{Thyroid disease cases}

We examined 381 cases of thyroid lesions from 376 patients which were treated surgically in the period between January
1995 to June 2011 at the King Abdulaziz University Hospital, Jeddah, and the King Faisal Specialist Hospital \& Research Center, Jeddah, or were referral/consultant cases from other regional hospitals. In five cases, tumor and non-tumor lesions from the same patient were evaluated in the study separately. Saudi Arabian nationality was reported for $61 \%$ of the patients and 27\% originated from other MENA (Middle East and North Africa) countries. A minority of patients originated from other world regions (8\%) or patient's nationality was not recorded (4\%). Histopathological diagnosis and staging of thyroid lesions was performed by an oncologic pathologist (JM) according to established criteria [11,12]. Cases were selected on basis of sample and data availability. With the exception of MTC [13] the most common, surgically treated thyroid diseases were included in the study, i.e. Hashimoto's thyroiditis, nodular goiters, hyperplastic lesions, FA, FTC (including Hurthle cell carcinomas), PTC $(>1 \mathrm{~cm})$, FVPTC $(>1 \mathrm{~cm})$, micro PTC $(\leq 1 \mathrm{~cm})$ and non-well differentiated TC (non-WDTC), The non-WDTC group comprised poorly differentiated insular variant PTC, combinatorial PTC/ ATC, and ATC. PTC $(>1 \mathrm{~cm})$ and FVPTC $(>1 \mathrm{~cm})$ are hereinafter referred as PTC and FVPTC, respectively.

Demographic and clinicopathological data were compiled from patients' files and included age, gender, family history of thyroid disorders/lesions, histological type, vessel (lymphatic, perineural, or vascular) invasion, capsular invasion, tumor extension, tumor size, multifocal tumors (including cases of subsequent partial thyroidectomies), lymph node involvement, distant metastasis (comprising radiologic findings), and tumor stage. Information to family history of thyroid disorders/lesions was voluntarily given by patients and regarded as positive if at least one first degree relative or two second degree relatives were affected. This study was approved by the ethical review boards of both institutions.

\section{Mutational screening}

Included in mutational analysis of BRAF exon 15 were specimens from primary thyroid lesions except in 15 cases in which only specimens from a recurrence or metastasis were available. A pathologist (JM) has reviewed each case and chose only specimens with not less than $70 \%$ of abnormal or tumor cells, respectively. Genomic DNA was extracted in the majority of cases from $10 \mu \mathrm{m}$ sections of formalin-fixed and paraffin-embedded (FFPE) material and using conventional xylene/ethanol treatment, overnight incubation with proteinase $\mathrm{K}$, and subsequent DNA purification utilizing the QIAmp DNA FFPE tissue kit (Qiagen, Hilden, Germany). In 116 cases native or fresh-frozen (FF) specimens were preserved and in these cases the QiAmp DNA mini kit was used for DNA purification. DNA concentration was measured with the Nanodrop device (Thermo Scientific, Wilmington, $\mathrm{DE})$.

The standard PCR protocol was performed as described earlier [13]. PCR products were checked by electrophoresis 
on $2 \%$ agarose gels. Purified PCR products were subjected to cycle sequence reactions using nested primers overlapping with the PCR primers and the BigDye Terminator V3.1 Cycle Sequencing kit (Applied Biosystems, Foster City, CA, USA). Purified sequencing products were finally resolved by capillary electrophoresis on an ABI PRISM 3130 Sequencer. Sequences were screened for BRAF exon 15 mutations using a combination of manual readout of electropherograms and the online NCBI's BLAST database [14].

\section{Statistical analysis}

Associations of demographic data, clinicopathological factors, and BRAF mutational status $\left(\mathrm{BRAF}^{\mathrm{wt}} v s . \mathrm{BRAF}^{\mathrm{mut}}\right)$ in histological types of TC were evaluated using the nonparametric Wilcoxon rank sum test or Fisher's exact test for contingency tables in the 2-sided configuration. P-value for accepting significance was $\mathrm{p}=0.05$. Statistical analysis was performed using the SPSS statistics 16 program (IBM Corp., New York, NY).

\section{Results}

The hotspot region in BRAF exon 15 was screened for mutations in 381 cases of cancerous and non-cancerous thyroid lesions. The case series comprised 10 Hashimoto`s thyroiditis lesions, 46 nodular goiters, 18 hyperplastic nodules, 69 FA, 115 PTC, 42 FVPTC, 56 micro PTC $(\leq 1 \mathrm{~cm}), 17$ FTC, and eight non-WDTC (2 insular PTC, 1 PTC/ATC, and 5 ATC) (Table 1). Mean age at diagnosis in non-malignant lesions ranged between 34.4 years in patients with hyperplastic nodules and 41.9 years in patients with goiter. In the TC group, mean age varied between 36.4 years in patients with micro PTC and 59 years in non-WDTC cases. Patients with micro PTC were on average 4 years younger than their PTC and FVPTC counterparts. Number of female cases predominated in all histological types. Of notice, female to male ratio was considerably higher in micro PTC than in PTC (6:1 vs. 2.3:1). Almost all micro PTC were classified as stage I tumors except two multifocal stage III tumors (follicular variant in one case) and one stage IV tumor (oncocytic variant) (Table 2). The majority of cases were stage I tumors in PTC (64\%), FVPTC (57\%), and FTC (65\%). Seven of eight nonWDTC cases were stage IV tumors and one was a stage II tumor. Follow-up period in TC patients ranged between 0 and 23 years (mean, 3.2 years; SD 3.2). Distant metastases were reported for PTC (15\%), FVPTC (17\%), FTC (6\%), and non-WDTC (50\%). Eight patients deceased from disease (age at first diagnosis, 49 to 78 years; mean age, 61 years); tumor progression in these patients was primarily confined to regional sites in two cases (1 PTC and 1 PTC with tall cell features metastasized as ATC), to distant sites in four cases (1 PTC, $1 \mathrm{FTC}, 1 \mathrm{PTC} / \mathrm{ATC}$ and $1 \mathrm{ATC}$ ) and to regional and distant sites in two cases (1 PTC and 1 FVPTC). The mean survival period of these patients was 3.9 years (SD 3.4).

Among non-malignant thyroid lesions one of 69 FA exhibited a BRAF mutation. In thyroid malignancies we identified BRAF mutations in 72 of 115 PTC (63\%) seven of $42(17 \%)$ FVPTC, 10 of 56 (18\%) micro PTC, one of 17 (6\%) FTC, and one of eight (13\%) non-WDTC (Table 1). Most of the BRAF mutations were the common point mutation in codon 600 leading to substitution of valine by glutamic acid (V600E). One PTC, FVPTC, and FTC each harbored a point mutation in codon 601 resulting in substitution of lysine by glutamic acid (K601E). The K601E mutation that is usually associated with FVPTC histology was identified in case of the FTC (Figure 1A, B) in FF as well as FFPE samples of the tumor. The K601E mutation in the PTC was detected in the primary tumor as well as in the regional progressive metastasis (Figure $1 C, D$ ). A yet undescribed BRAF mutation was detected in different sections of a FA (Figure 1E, F). This 3base-pair deletion at codon 599 erases threonine 599 (T599del) (Figure 2A). A very rare BRAF 3-base-pair insertion at codon 599, resulting in duplication of threonine 599 (T599dup), was detected in a multifocal, stage III PTC that developed distant metastases within 4.5 years (Figure 2B). Both, T599del and T599dup were confined to the tumor.

Table 1 Demographic and BRAF mutational survey in thyroid lesions

\begin{tabular}{|c|c|c|c|c|c|c|c|}
\hline \multirow[t]{2}{*}{ Thyroid lesion } & \multirow[t]{2}{*}{ Number } & \multirow{2}{*}{$\begin{array}{c}\text { Age at diagnosis } \\
\text { (years, SD) }\end{array}$} & \multirow{2}{*}{$\begin{array}{c}\text { Female/male } \\
\text { ratio }\end{array}$} & \multicolumn{2}{|c|}{ Family history } & \multicolumn{2}{|r|}{ BRAF status $^{1}$} \\
\hline & & & & neg & pos & wt & mut \\
\hline Thyroiditis & 10 & $36.7 \pm 12.5$ & 10:0 & 5 & 2 & 10 & 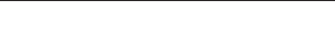 \\
\hline Goiter & 46 & $41.9 \pm 11.4$ & 2.3:1 & 19 & 5 & 46 & \\
\hline Hyperplastic & 18 & $34.4 \pm 12.5$ & $8: 1$ & 8 & 0 & 18 & \\
\hline FA & 69 & $38.4 \pm 13.1$ & $3: 1$ & 11 & 2 & 68 & 1 T599de \\
\hline FTC & 17 & $40.4 \pm 16.7$ & $3.3: 1$ & 2 & 2 & 16 & $1 \mathrm{~K} 601$ \\
\hline PTC & 115 & $41.1 \pm 15.5$ & $2.3: 1$ & 27 & 4 & 43 & 1 T599insT, 70 V600E, 1 K601 \\
\hline FVPTC & 42 & $40.5 \pm 15.6$ & $5: 1$ & 9 & 4 & 35 & $6 \mathrm{~V} 600 \mathrm{E}, 1 \mathrm{~K} 601$ \\
\hline Micro PTC & 56 & $36.4 \pm 13.5$ & $6: 1$ & 10 & 4 & 46 & 10 V600 \\
\hline Non-WDTC & 8 & $59.0 \pm 10.1$ & $7: 1$ & 1 & 1 & 7 & $1 \mathrm{~V} 600$ \\
\hline
\end{tabular}

${ }^{1} \mathrm{Wt}$, BRAF wild type; mut, BRAF mutated. 
Table 2 Distribution of tumor stages and BRAF mutations in thyroid malignancies

\begin{tabular}{cccccc}
\hline Stage & PTC & FVPTC & micro PTC & FTC & non-WDTC \\
\hline I & $74[31 / 43]$ & $24[19 / 5]$ & $53[45 / 9]$ & $11[10 / 1]$ & 0 \\
II & $9[4 / 5]$ & $6[4 / 2]$ & 0 & 2 & $1[0 / 1]$ \\
III & $15[3 / 12]$ & 7 & $2[1 / 1]$ & 3 & 0 \\
IV & $15[5 / 10]$ & 4 & 1 & 1 & 7 \\
Unknown & $2[0 / 2]$ & 1 & & & \\
\hline BRAFWt/BRAF &
\end{tabular}

Tumor size $\geq 4 \mathrm{~cm}(\mathrm{P}=0.018)$, vessel invasion $(\mathrm{P}=0.004)$, and distant metastasis $(\mathrm{P}=0.001)$ were significantly more common in PTC patients aged 45 years and older (Table 3 ). Patients with $\mathrm{BRAF}^{\mathrm{wt}}$ PTC were on average younger than those with BRAF ${ }^{\text {mut }}$ PTC (36.6 years vs. 43.8 years; $\mathrm{P}=0.025$ ). $\mathrm{LN}$ involvement in PTC associated with tumor size $(\mathrm{P}=0.044)$, and vessel invasion $(\mathrm{P}=0.013)$. Tumor size was slightly, but not significantly, larger in $\mathrm{BRAF}^{\mathrm{mut}}$ than in $\mathrm{BRAF}^{\mathrm{wt}}$ PTC (3.6 cm vs. $2.9 \mathrm{~cm}$; determined for 87 cases). Considering all histological types of TC as a group, i.e. PTC, FVPTC, micro PTC, FTC, and non-WDTC, family history of thyroid disease positively correlated with capsular invasion $(\mathrm{P}=0.025)$.

\section{Discussion}

This study is one of the first comprehensive surveys on BRAF mutations in different types of thyroid diseases carried out so far in the MENA region. In general, frequency, distribution, and risk assessment of BRAF mutations in TC differ between studies emphasizing that the genetic etiology
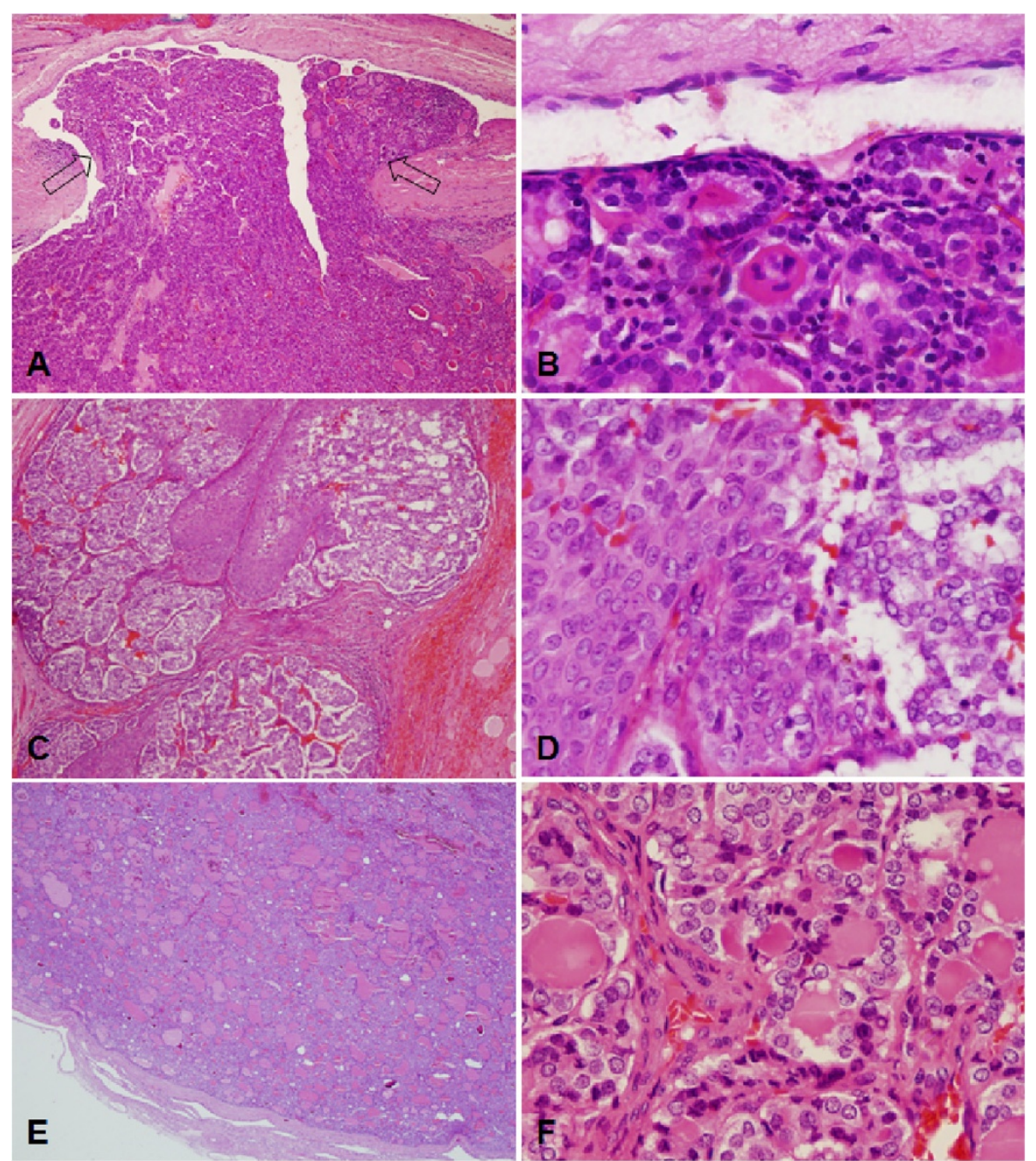

Figure 1 Hematoxylin and eosin stains of thyroid cancer cases harboring new or rare BRAF mutations. Left column, original magnification $40 \mathrm{x}$; right column, original magnification $400 \mathrm{x}$. A and B, FTC with a BRAF K601E mutation. Case has a single minimally invasive focus, marked by arrows. C and D, PTC with BRAF K601E mutation. The recurrence of the case involves subcutaneous tissue and skeletal muscle. Conventional PTC and squamous components are intermixed. $\mathbf{E}$ and $\mathbf{F}$, FA harboring a deletion of BRAF codon 599. The tumor presents as a solitary encapsulated nodule and misses nuclear features of PTC. 


\section{$\begin{array}{llllll}\text { A wt } & \text { L597 } & \text { A598 } & \text { T599 } & \text { V600 } & \text { K601 } \\ \text { del } & \text { L597 } & \text { A598 } & \text { V600 } & \text { K601 } & \text { S602 }\end{array}$

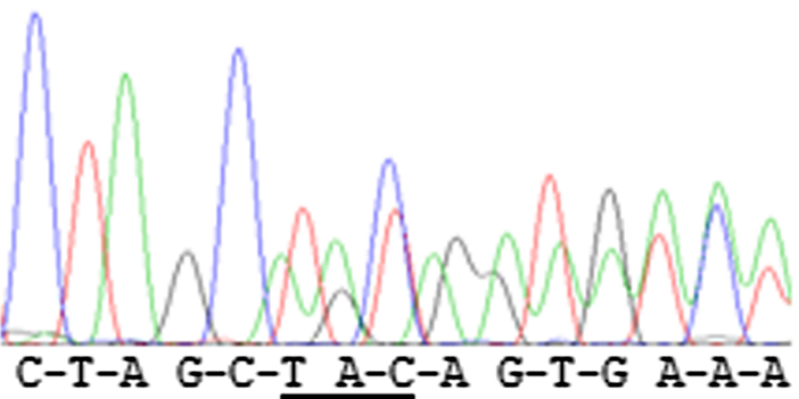 \\ we $\mathrm{C}-\mathrm{T}-\mathrm{A}$ G-C-T $\mathrm{A}-\mathrm{C}-\mathrm{A}$ G-T-G A-A-A \\ del C-T-A G-C-A G-T-G A-A-A T-C-T}
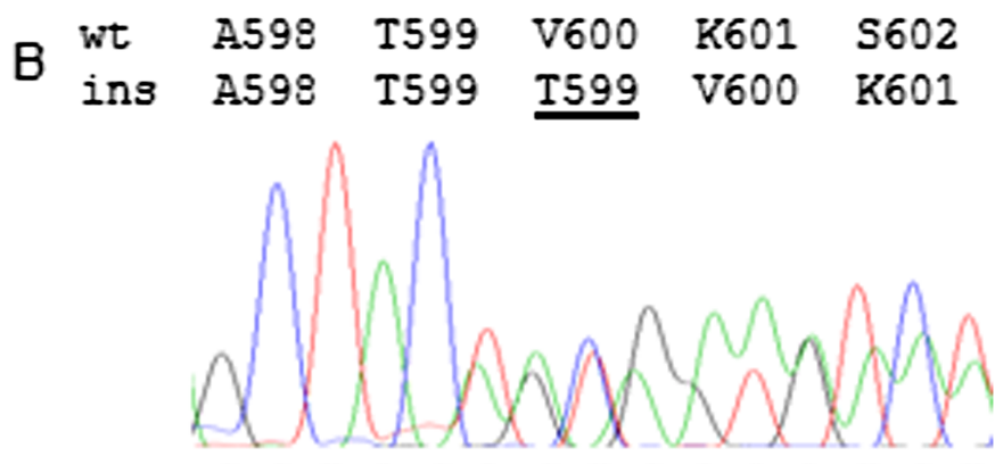

wt $\mathrm{G}-\mathrm{C}-\mathrm{T}$ A-C-A G-T-G A-A-A T-C-T

ins $\mathrm{G}-\mathrm{C}-\mathrm{T} \mathrm{A}-\mathrm{C}-\mathrm{T} \mathrm{A}-\mathrm{C}-\mathrm{A}$ G-T-G $\mathrm{A}-\mathrm{A}-\mathrm{A}$

Figure 2 New and sporadic BRAF mutations in thyroid neoplasms. A, a new 3-base-pair deletion at codon 599 identified in a FA resulting in deletion of threonine 599 (p.T599del). B, a rare 3-base pair insertion at codon 599 found in an aggressive PTC resulting in duplication of threonine 599 (p.T599dup). Deleted and inserted nucleotides and codons are underlined.

of $\mathrm{TC}$ is complex and varies between the populations studied $[15,16]$. The overall incidence of BRAF mutations identified in our series and the gradation of frequency of BRAF mutations between PTC and FVPTC are similar to those displayed in other studies [17]. The detection rate of

Table 3 Significant associations of demographic/ clinocopathological data and BRAF mutations in thyroid malignancies

\begin{tabular}{lccc}
\hline Factors $^{\mathbf{1}}$ & Histology & P value & Test $^{\mathbf{2}}$ \\
\hline Age $\geq 45$ years vs. tumor size $\geq 4 \mathrm{~cm}[87]$ & PTC & 0.018 & FET \\
Age $\geq 45$ years vs. vessel invasion [113] & PTC & 0.004 & FET \\
Age $\geq 45$ years vs. distant metastasis [115] & PTC & 0.001 & FET \\
LN + vs. tumor size $\geq 4$ cm [43] & PTC & 0.044 & FET \\
LN + vs. vessel invasion [59] & PTC & 0.013 & FET \\
Increasing age vs. BRAF ${ }^{\text {mut }}[115]$ & PTC & 0.025 & MWU \\
Family history vs. capsular invasion [64] & TC & 0.025 & FET \\
\hline
\end{tabular}

${ }^{1}$ Number of cases included in analysis in brackets; ${ }^{2} \mathrm{FET}$, Fisher's exact test; MWU, Mann-Whitney $U$ test.
$\mathrm{BRAF}^{\text {mut }}$ in PTC varies between approximately 30\% and 85\% while for FVPTC the rate varies between $0 \%$ and $35 \%$ with on average $\sim 15 \%$ measured across different studies [7,17-20]. Only a few studies have revealed a similar large discrepancy in age distribution between $\mathrm{BRAF}^{\mathrm{wt}}$ and BRAF $^{\text {mut }}$ tumors as we did [21-23] whereas other investigators did not reveal any significant difference and a metanalysis on the clinicopathological impact of BRAF mutations in PTC could not compile age specific differences across six evaluated studies $[17,24]$. The overall young age distribution in our series can be attributed to the young population structure of the region.

In general, significance of the BRAF mutational status in regard to clinicopathological features and clinical treatment of the disease is still matter of debate although a trend of BRAF mutation with aggressive tumor markers like vessel invasion, capsular invasion, tumor extension, or LN metastases has been revealed in a number of studies $[10,16,17,24,25]$. We attribute the low clinicopathological significance of BRAF mutations in our series to the fact that this driver 
mutation is already present in the majority of low stage PTC. The specific correlation of BRAF mutations with poor outcome seems to become more evident in long-term follow-up studies $[23,26]$. In contrast, BRAF mutations in micro PTC are less likely to correlate with clinicopathological factors [27]. These data coincide with our findings. Our observation that capsular invasion correlates with family history of TC warrants further exploration to sustain these findings and to identify possible genetic predisposition effects in affected individuals.

The K601E mutation, identified in our series in one case each of FTC, FVFTC, and PTC is known to be associated with FVPTC histology [9,24,28-30]. BRAF mutations in TC others than the V600E mutation are commonly associated with non-aggressive tumors [31]; however, association of T599dup and K601E mutations with advanced TC has been reported $[32,33]$.

BRAF mutations in FA have only been identified so far sporadically and are almost K601E mutations which FA have in common with their follicular malignant counterparts $[30,34,35]$. The novel T599del mutation detected in a FA in our series affects one of the two critical phosphorylation sites (T599 and S602) in the activation domain encoded by exon 15. Phosphorylation of these residues disrupts the hydrophobic association of the phosphate-binding loop (P loop) with the activation loop (A loop) $[8,36]$. It can be hypothesized that deletion of codon 599 confers a conformational change in a similar manner as phosphorylation of T599 which results in destabilization of the inactive conformation and switch into the active configuration. Further studies have to reveal if the T599del is functionally linked to FA development which would separate it from other BRAF mutations evolving in TC.

The T599dup identified in an aggressive PTC in our series has been recently uncovered as an activation mutation in pilocytic astrocytomas and a small number of ATC with tall cell features [33,37,38]. The T599dup exhibited an elevated kinase activity in transfection assays similarly to V600E [38]. The authors supposed that addition of an extra amino acid residue at this position rather than addition of a second threonine phosphorylation site results in disruption of the inhibitory conformation of the activation loop. Of notice, a considerable number of sporadic mutations in BRAF exon 15 affects or co-affects codon 599 in TC [24,39-43]. These mutations tested so far exhibit elevated kinase activity emphasizing the critical cofunction of codon 599 in the BRAF activation process $[8,38,40,42]$.

\section{Conclusions}

Significant findings of our study include new and rare BRAF mutations in malignant and non-malignant thyroid tumors that in cases of T599dup and K601E seem to confer a certain risk for progressive TC. Other notable findings include correlation of capsular invasion with family history of
TC whereas BRAF mutational status disclosed only limited associations with clinicopathological factors in our series.

\section{Abbreviations}

ATC: Anaplastic TC; BRAF ${ }^{\text {mut }}$ : BRAF mutation; BRAF ${ }^{\text {wt }}$ : BRAF wild type; FA: Follicular adenoma(s); FF: Fresh-frozen; FFPE: Formalin-fixed and paraffinembedded; FTC: Follicular TC; FVPTC: Follicular variant PTC; LN: Lymph node; MAPK: Mitogen activated protein kinase; MENA: Middle East and North Africa; micro PTC: Microcarcinoma of PTC; MTC: Medullary TC; non-WDTC: Non-well differentiated TC; PTC: Papillary TC; TC: Thyroid carcinoma(s).

\section{Competing interests}

The authors declare that they have no competing interests.

\section{Authors' contributions}

$\mathrm{SS}, \mathrm{EH}, \mathrm{MG}$, and ZM made substantial contributions to the conception and design of the study. RA performed sequence analyses of PTC. KG, OAH, and AA were responsible for surgeries, oversight of clinical databases and contributed to the conception and design of the study. SS, HS, HA, and JM performed histological examinations. HJS performed statistical analysis and had general oversight of the study. HJS, JM, and MQ interpreted data and drafted the manuscript. All authors read and approved the final manuscript.

\section{Acknowledgments}

We thank Shireen Hussain, Nadia Bagation, and Ibtesam Baglab for excellent technical assistance. This study was supported by King Abdulaziz City for Science and Technology (KACST) grants 09-BI0707-03 and 09-BIO820-03.

\section{Author details}

${ }^{1}$ Center of Excellence in Genomic Medicine Research, King Abdulaziz University, Jeddah, Saudi Arabia. ${ }^{2}$ Department of Pathology, Faculty of Medicine, King Abdulaziz University, Jeddah, Saudi Arabia. ${ }^{3}$ Department of Pathology, King Faisal Specialist Hospital and Research Center, Jeddah, Saudi Arabia. ${ }^{4}$ Department of Biochemistry, King Abdulaziz University, Jeddah, Saudi Arabia. ${ }^{5}$ Department of Surgery, Faculty of Medicine, King Abdulaziz University, Jeddah, Saudi Arabia. ${ }^{6}$ Department of Surgery, King Faisal Specialist Hospital and Research Center, Jeddah, Saudi Arabia.

Received: 3 August 2012 Accepted: 24 August 2012

Published: 27 August 2012

\section{References}

1. Jemal A, Bray F, Center MM, Ferlay J, Ward E, Forman D: Global cancer statistics. CA Cancer J Clin 2011, 61:69-90.

2. Hundahl SA, Cady B, Cunningham MP, Mazzaferri E, McKee RF, Rosai J, Shah JP, Fremgen AM, Stewart AK, Holzer S: Initial results from a prospective cohort study of 5583 cases of thyroid carcinoma treated in the united states during 1996. U.S. and German Thyroid Cancer Study Group. An American College of Surgeons Commission on Cancer Patient Care Evaluation study. Cancer 2000, 89:202-217.

3. Ministry of Health: Riyadh (KSA): Annual Cancer Incidence Report, Saudi Arabia. http://www.scr.org.sa/index.php?lang=ar; http://www.scr.org.sa/ reports/SCR2007.pdf.

4. Smallridge RC, Marlow LA, Copland JA: Anaplastic thyroid cancer: molecular pathogenesis and emerging therapies. Endocr Relat Cancer 2009, 16:17-44.

5. Qari FA: Pattern of thyroid malignancy at a University Hospital in Western Saudi Arabia. Saudi Med J 2004, 25:866-870.

6. Davies H, Bignell GR, Cox C, Stephens P, Edkins S, Clegg S, Teague J, Woffendin H, Garnett MJ, Bottomley W, et al: Mutations of the BRAF gene in human cancer. Nature 2002, 417:949-954.

7. Xing M, Westra WH, Tufano RP, Cohen Y, Rosenbaum E, Rhoden KJ, Carson KA, Vasko V, Larin A, Tallini G, et al: BRAF mutation predicts a poorer clinical prognosis for papillary thyroid cancer. J Clin Endocrinol Metab 2005, 90:6373-6379.

8. Wan PT, Garnett MJ, Roe SM, Lee S, Niculescu-Duvaz D, Good VM, Jones CM, Marshall CJ, Springer CJ, Barford D, Marais R: Mechanism of activation of the RAF-ERK signaling pathway by oncogenic mutations of B-RAF. Cell 2004, 116:855-867.

9. Trovisco V, Vieira de Castro I, Soares P, Maximo V, Silva P, Magalhaes J, Abrosimov A, Guiu XM, Sobrinho-Simoes M: BRAF mutations are 
associated with some histological types of papillary thyroid carcinoma. J Pathol 2004, 202:247-251.

10. Nucera C, Goldfarb M, Hodin R, Parangi S: Role of B-Raf(V600E) in differentiated thyroid cancer and preclinical validation of compounds against B-Raf(V600E). Biochim Biophys Acta 2009, 1795:152-161.

11. Thyroid. In AJCC Cancer Staging Manual. 7th edition. Edited by Edge SB, Byrd DR, Compton CC, Fritz AG, Greene FL, Trotti A. New York: Springer; 2010:87-96.

12. DeLellis RA, Williams ED: Thyroid and parathyroid tumors. In Pathology and genetics of tumours of endocrine organs (IARC WHO classification of tumours). Edited by DeLellis RA, Lloyd RV, Heitz PU, Eng C. Lyons: IARC Press; 2004: $51-56$.

13. Schulten HJ, Al-Maghrabi J, Al-Ghamdi K, Salama S, Al-Muhayawi S, Chaudhary A, Hamour O, Abuzenadah A, Gari M, Al-Qahtani M: Mutational screening of RET, HRAS, KRAS, NRAS, BRAF, AKT1, and CTNNB1 in medullary thyroid carcinoma. Anticancer Res 2011, 31:4179-4183.

14. http://blast.ncbi.nlm.nih.gov/Blast.cgi.

15. Abubaker J, Jehan Z, Bavi P, Sultana M, Al-Harbi S, Ibrahim M, Al-Nuaim A, Ahmed M, Amin T, Al-Fehaily M, et al: Clinicopathological analysis of papillary thyroid cancer with PIK3CA alterations in a Middle Eastern population. J Clin Endocrinol Metab 2008, 93:611-618.

16. Frasca F, Nucera C, Pellegriti G, Gangemi P, Attard M, Stella M, Loda M, Vella $V$, Giordano C, Trimarchi F, et al: BRAF(V600E) mutation and the biology of papillary thyroid cancer. Endocr Relat Cancer 2008, 15:191-205.

17. Lee JH, Lee ES, Kim YS: Clinicopathologic significance of BRAF V600E mutation in papillary carcinomas of the thyroid: a meta-analysis. Cancer 2007, 110:38-46.

18. Fugazzola L, Puxeddu E, Avenia N, Romei C, Cirello V, Cavaliere A, Faviana P, Mannavola D, Moretti S, Rossi S, et al: Correlation between B-RAFV600E mutation and clinico-pathologic parameters in papillary thyroid carcinoma: data from a multicentric Italian study and review of the literature. Endocr Relat Cancer 2006, 13:455-464.

19. Costa AM, Herrero A, Fresno MF, Heymann J, Alvarez JA, Cameselle-Teijeiro J, Garcia-Rostan G: BRAF mutation associated with other genetic events identifies a subset of aggressive papillary thyroid carcinoma. Clin Endocrinol (Oxf) 2008, 68:618-634

20. Kebebew E, Weng J, Bauer J, Ranvier G, Clark OH, Duh QY, Shibru D, Bastian $B$, Griffin A: The prevalence and prognostic value of BRAF mutation in thyroid cancer. Ann Surg 2007, 246:466-470. discussion 470-461.

21. Nikiforova MN, Kimura ET, Gandhi M, Biddinger PW, Knauf JA, Basolo F, Zhu Z, Giannini R, Salvatore G, Fusco A, et al: BRAF mutations in thyroid tumors are restricted to papillary carcinomas and anaplastic or poorly differentiated carcinomas arising from papillary carcinomas. J Clin Endocrinol Metab 2003, 88:5399-5404.

22. Kim KH, Kang DW, Kim SH, Seong IO, Kang DY: Mutations of the BRAF gene in papillary thyroid carcinoma in a Korean population. Yonsei Med $J$ 2004, 45:818-821.

23. Elisei R, Ugolini C, Viola D, Lupi C, Biagini A, Giannini R, Romei C, Miccoli P, Pinchera A, Basolo F: BRAF(V600E) mutation and outcome of patients with papillary thyroid carcinoma: a 15-year median follow-up study. J Clin Endocrinol Metab 2008, 93:3943-3949.

24. Lupi C, Giannini R, Ugolini C, Proietti A, Berti P, Minuto M, Materazzi G, Elisei R, Santoro M, Miccoli P, Basolo F: Association of BRAF V600E mutation with poor clinicopathological outcomes in 500 consecutive cases of papillary thyroid carcinoma. J Clin Endocrinol Metab 2007, 92:4085-4090.

25. So YK, Son YI, Park JY, Baek CH, Jeong HS, Chung MK: Preoperative BRAF mutation has different predictive values for lymph node metastasis according to tumor size. Otolaryngol Head Neck Surg 2011, 145:422-427.

26. Namba H, Nakashima M, Hayashi T, Hayashida N, Maeda S, Rogounovitch TI, Ohtsuru A, Saenko VA, Kanematsu T, Yamashita S: Clinical implication of hot spot BRAF mutation, V599E, in papillary thyroid cancers. J Clin Endocrinol Metab 2003, 88:4393-4397.

27. Kim TY, Kim WB, Song JY, Rhee YS, Gong G, Cho YM, Kim SY, Kim SC, Hong SJ, Shong YK: The BRAF mutation is not associated with poor prognostic factors in Korean patients with conventional papillary thyroid microcarcinoma. Clin Endocrinol (Oxf) 2005, 63:588-593.

28. Frau DV, Lai ML, Caria P, Dettori T, Coni P, Faa G, Morandi L, Tallini G, Vanni R: Trisomy 17 as a marker for a subset of noninvasive thyroid nodules with focal features of papillary carcinoma: cytogenetic and molecular analysis of 62 cases and correlation with histological findings. J Clin Endocrinol Metab 2008, 93:177-181.
29. Castro P, Rebocho AP, Soares RJ, Magalhaes J, Roque L, Trovisco V, Vieira de Castro I, Cardoso-de-Oliveira M, Fonseca E, Soares P, Sobrinho-Simoes M: PAX8-PPARgamma rearrangement is frequently detected in the follicular variant of papillary thyroid carcinoma. J Clin Endocrinol Metab 2006, 91:213-220

30. Trovisco V, Soares P, Preto A, de Castro IV, Lima J, Castro P, Maximo V, Botelho T, Moreira S, Meireles AM, et al: Type and prevalence of BRAF mutations are closely associated with papillary thyroid carcinoma histotype and patients' age but not with tumour aggressiveness. Virchows Arch 2005, 446:589-595.

31. Nikiforov YE, Nikiforova MN: Molecular genetics and diagnosis of thyroid cancer. Nat Rev Endocrinol 2011, 7:569-580.

32. Kloos RT, Ringel MD, Knopp MV, Hall NC, King M, Stevens R, Liang J, Wakely PE Jr, Vasko W, Saji M, et al: Phase II trial of sorafenib in metastatic thyroid cancer. J Clin Oncol 2009, 27:1675-1684.

33. Gauchotte G, Philippe C, Lacomme S, Leotard B, Wissler MP, Allou L, Toussaint B, Klein M, Vignaud JM, Bressenot A: BRAF, p53 and SOX2 in anaplastic thyroid carcinoma: evidence for multistep carcinogenesis. Pathology 2011, 43:447-452.

34. Soares P, Trovisco V, Rocha AS, Lima J, Castro P, Preto A, Maximo V, Botelho T, Seruca R, Sobrinho-Simoes M: BRAF mutations and RET/PTC rearrangements are alternative events in the etiopathogenesis of PTC. Oncogene 2003, 22:4578-4580.

35. Lima J, Trovisco V, Soares P, Maximo V, Magalhaes J, Salvatore G, Santoro M, Bogdanova T, Tronko M, Abrosimov A, et al: BRAF mutations are not a major event in post-Chernobyl childhood thyroid carcinomas. J Clin Endocrinol Metab 2004, 89:4267-4271.

36. Roskoski R Jr: RAF protein-serine/threonine kinases: structure and regulation. Biochem Biophys Res Commun 2010, 399:313-317.

37. Jones DT, Kocialkowski S, Liu L, Pearson DM, Ichimura K, Collins VP: Oncogenic RAF1 rearrangement and a novel BRAF mutation as alternatives to KIAA1549:BRAF fusion in activating the MAPK pathway in pilocytic astrocytoma. Oncogene 2009, 28:2119-2123.

38. Eisenhardt $A E$, Olbrich $H$, Roring $M$, Janzarik W, Anh $T N$, Cin $H$, Remke $M$, Witt $\mathrm{H}$, Korshunov A, Pfister SM, et al: Functional characterization of a BRAF insertion mutant associated with pilocytic astrocytoma. Int I Cancer 2011, 129:2297-2303.

39. Carta C, Moretti S, Passeri L, Barbi F, Avenia N, Cavaliere A, Monacelli M, Macchiarulo A, Santeusanio F, Tartaglia M, Puxeddu E: Genotyping of an Italian papillary thyroid carcinoma cohort revealed high prevalence of BRAF mutations, absence of RAS mutations and allowed the detection of a new mutation of BRAF oncoprotein (BRAF(V599|ns)). Clin Endocrinol (Oxf) 2006, 64:105-109

40. Moretti S, Macchiarulo A, De Falco V, Avenia N, Barbi F, Carta C, Cavaliere A, Melillo RM, Passeri L, Santeusanio F, et al: Biochemical and molecular characterization of the novel BRAF(V599Ins) mutation detected in a classic papillary thyroid carcinoma. Oncogene 2006, 25:4235-4240.

41. Hou P, Liu D, Xing M: Functional characterization of the T1799-1801del and A1799-1816ins BRAF mutations in papillary thyroid cancer. Cell Cycle 2007, 6:377-379.

42. De Falco V, Giannini R, Tamburrino A, Ugolini C, Lupi C, Puxeddu E, Santoro $M$, Basolo F: Functional characterization of the novel T5991-VKSRdel BRAF mutation in a follicular variant papillary thyroid carcinoma. J Clin Endocrinol Metab 2008, 93:4398-4402.

43. Chiosea S, Nikiforova M, Zuo H, Ogilvie J, Gandhi M, Seethala RR, Ohori NP, Nikiforov $Y$ : A novel complex BRAF mutation detected in a solid variant of papillary thyroid carcinoma. Endocr Pathol 2009, 20:122-126.

doi:10.1186/1897-4287-10-10

Cite this article as: Schulten et al: BRAF mutations in thyroid tumors from an ethnically diverse group. Hereditary Cancer in Clinical Practice 2012 10:10. 